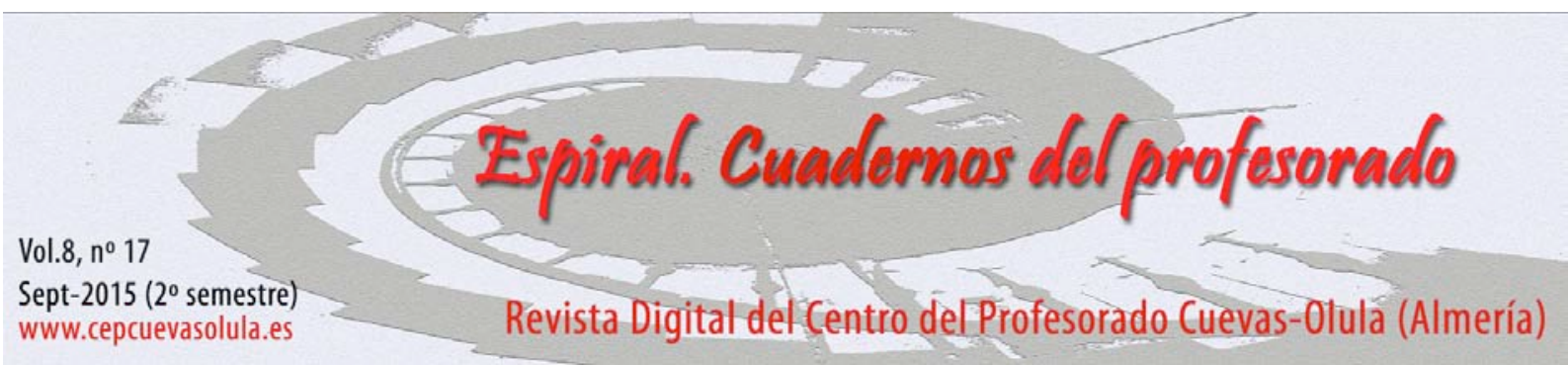

\title{
RENOVACIÓN METODOLÓGICA EN EL ESPACIO EUROPEO DE EDUCACIÓN SUPERIOR
}

\author{
Methodological renewal in the European Higher Education Area \\ Dimitrinka G. Níkleva ${ }^{(1)}$ y Francisco J. Rodríguez Muñoz ${ }^{(2)}$ \\ (1) Universidad de Granada, España \\ (2) Universidad de Almería, España
}

\begin{abstract}
RESUMEN: El presente estudio está dedicado al Espacio Europeo de Educación Superior (EEES) y a las novedades que ha supuesto para la universidad española. Además, se pretende dejar constancia de algunos logros y deficiencias. El objeto principal del estudio es la renovación metodológica en el marco del EEES: el aprendizaje a lo largo de la vida, el sistema de admisión de estudiantes, la combinación de métodos de enseñanza-aprendizaje, la evaluación formativa de los estudiantes, las tutorías (características, dimensiones y niveles de intervención en la tutoría, tipos de tutoría, roles y competencias del profesor tutor, la opinión de los propios estudiantes), etc. El estudio finaliza con una reflexión crítica sobre el EEES, fruto de la preocupación de distintos sectores relacionados con el ámbito de la enseñanza. Existe una preocupación por el valor que cobraría la cantidad en lugar de la calidad, los resultados en lugar del proceso, los intereses económicos, la competitividad y las demandas del mercado en lugar de la formación integral del individuo. Además, es necesario aumentar la relevancia social de la formación y de la investigación, la eficiencia de la educación superior, la autonomía de las universidades y su diferenciación, así como el prestigio internacional de las universidades españolas.
\end{abstract}

Palabras clave: Espacio Europeo de Educación Superior, renovación metodológica, aprendizaje a lo largo de la vida, evaluación, tutorías, reflexión crítica.

ABSTRACT: The present study is devoted to the European Higher Education Area (EHEA) and to the innovations that it has brought to the Spanish university. In addition, it aims at showing some achievements and shortcomings. The main purpose of the study is the methodological reform within the EHEA context: learning throughout life, the student admission system, the combination of methods of teaching and learning, formative assessment of students, tutorial classes (characteristics, dimensions and levels of intervention in mentoring, types of academic supervision, roles and responsibilities of the tutor, the opinion of the own students), etc. The study concludes with a critical reflection on the EHEA, as a result of the concern of different sectors related to the field of education. It is worrying the fact that quantity could be more valuable than quality, the results rather than the process, economic interests, competitiveness and market demands rather than the integral education of individuals. Furthermore, it is necessary to increase the social relevance of training and research, the efficiency of higher education, university autonomy and differentiation, as well as the international prestige of Spanish universities.

Key words: European Higher Education Area, methodological reform, learning throughout life, formative assessment of students, tutorial classes, critical reflection. 
Níkleva, D. G. y Rodríguez Muñoz, F. (2015). Renovación metodológica en el Espacio Europeo de Educación Superior. Espiral. Cuadernos del Profesorado, 8(17), 3-15. Disponible en: http://www.cepcuevasolula.es/espiral.

Fecha de recepción: 08/04/2015

Fecha de aceptación: 17/07/2015
Enviar correspondencia a: nikleva@ugr.es

\section{1.- INTRODUCCIÓN}

En las últimas décadas la institución universitaria ha seguido desarrollando con más o menos acierto la función básica de ser vehículo de la continuidad cultural y de la transformación social. Los últimos cambios, sin ser recientes, derivan del hecho de que actualmente la enseñanza universitaria en Europa está enmarcada en el Espacio Europeo de Educación Superior (EEES) y en el Plan Bolonia.

Dedicamos el presente estudio a esta última etapa y a las novedades que ha supuesto para la universidad española para dejar constancia de algunos logros y deficiencias.

Partiendo de la convicción de que la labor docente debe ser una labor constructiva que crea puentes entre la vida universitaria y la vida social y profesional, resulta necesario recordar que en este camino todos los elementos del currículo deben ser actualizados para responder a la demanda social. La nueva situación exige una revisión de los cometidos asignados a la Universidad, de cara a su reconstrucción como organización e institución social.

Debemos considerar que la especialización tiene un desarrollo vertiginoso en la actualidad. De las dos grandes áreas la educación griega clásica, el trivium y el cuadrivium, se ha pasado a una enorme variedad de áreas de conocimiento. De este cambio deriva la necesidad de profesionalización en las titulaciones universitarias que es una de las claves del Plan Bolonia. De ahí, uno de los ejes más importantes en el marco de la convergencia europea quizás sea el hecho de tomar las competencias profesionales específicas y las competencias genéricas y transversales como punto de partida para la elaboración de cada programa docente. Obviamente, esto conlleva una renovación metodológica, necesaria para conseguir los nuevos retos. Y para diseñar una nueva metodología hay que partir de la convicción de que (Contreras González, 2007, 8):

la información no puede seguir pasando de los papeles del profesor a los papeles del estudiante sin pasar por la cabeza de ninguno de los dos. Además, hoy es más importante saber dónde está la información y saber procesarla de forma crítica que disponer de ella de forma enciclopédica, y desde luego, esa capacidad no emerge de forma espontánea en nuestros estudiantes, es preciso construirla de forma consciente. Por ello, otro cambio relevante es el de la necesaria variedad de actividades formativas orientadas hacia la adquisición de competencias procedimentales y al autoaprendizaje.

En este estudio nos vamos a centrar en la metodología, o sea, en el cómo del proceso de enseñanza-aprendizaje y en la renovación que ha supuesto en el EEES.

\section{2.- APRENDIZAJE A LO LARGO DE LA VIDA}

La renovación metodológica está enmarcada en una sociedad del conocimiento y la información, donde uno de los aspectos fundamentales es la velocidad con la que cambian los conocimientos y de allí, la imposibilidad de proporcionar una formación inicial a los estudiantes que sea válida para toda su vida profesional. Por eso, en esta sociedad tan dinámica social y económicamente se hace hincapié en el aprendizaje durante toda la vida. La consecuencia de ello es pasar de un modelo educativo, basado en la enseñanza o el aprendizaje de conocimientos, a otro, basado en el aprender a aprender. De esta manera, el alumno será el protagonista absoluto de su proceso de aprendizaje. Evidentemente, el papel del docente como orientador y guía sigue siendo muy importante, pero es ahora cuando el estudiante y su labor universitaria se convierten en núcleos del nuevo modelo educativo. 
Podríamos definir el aprendizaje a lo largo de toda la vida como un proceso formativo continuo que ayuda a los individuos a resolver las necesidades cambiantes que la vida personal y profesional les van presentando (López Yánez y Rodríguez López, 2007).

Hemos de tener en cuenta también que el aprendizaje es un proceso constructivo, que se optimiza cuando el material aprendido es útil y significativo para el individuo que lo aprende y cuando este se compromete de modo activo en la construcción de su propia comprensión y conocimiento al conectar lo que aprende con sus experiencias y conocimientos previos (McCombs y Whisler, 2000).

Todos los estudiantes tienen capacidad natural para aprender, $\mathrm{y}$, por tanto, también de dirigir su propio proceso de aprendizaje. Las diferencias entre los alumnos se dan en las competencias generales y específicas, en su estado emocional y la comprensión de las relaciones sociales que se generan en los grupos. El aprendizaje se optimiza en entornos positivos, de relaciones afectivas. El alumno necesita sentirse conocido, apreciado, comprendido, respetado y respaldado. Por ello, conviene que los profesores consulten a los alumnos a la hora de plantear los objetivos y los relacionen con situaciones cotidianas.

Se trata de un nuevo enfoque que se fundamenta en tres principios:

1) Mayor implicación y autonomía del estudiante.

2) Utilización de metodologías más activas: casos prácticos, trabajo en equipo, tutorías, seminarios, tecnologías multimedia, etc.

3) El papel del docente es el de generador de entorno de aprendizajes que estimulen a los alumnos.

La Universidad, en palabras de Imbernón (1999), debe abandonar la concepción decimonónica que ha quedado totalmente obsoleta y debe asumir una nueva cultura, tanto en la forma como en el contenido. El auge de la nuevas tecnologías, la especialización continua de la ciencia, la crítica al método científico tradicional, el concepto de ciencia, las nuevas actitudes sociales, la imprescindible formación continua de las personas, los nuevos medios formativos, etc., son, algunos de los aspectos, entre otros, que demandan otro tipo de Universidad que sepa dar respuesta a la demanda social.

Tomás (2000) destaca los siguientes motores de cambio que pueden ayudar a actualizar las respuestas que ofrece la Universidad como institución:

- La tecnología, los instrumentos informáticos y el acceso y exceso de información.

- El acceso generalizado de estudiantes a la Universidad.

- La creciente necesidad de formación continua y la transformación de la función docente.

- La nueva manera de entender la gestión.

- La realización de investigaciones, cada vez más multidisciplinares y grupales.

- Los intercambios con otros países europeos, cada vez más frecuentes e intensos.

Las nuevas exigencias se traducen en cambios que afectan tanto al contenido de la enseñanza como a su orientación. Marcelo (2001) presenta una aproximación a estos cambios en el siguiente cuadro (Tabla 1).

A pesar de los intentos de hacer efectivos los cambios en el marco europeo de convergencia, autores como Arranz (2008), a través de estudios realizados en la Universidad de Zaragoza, consideran que en el ámbito universitario se observan todavía prácticas que responden a la yuxtaposición de producciones, en las cuales cada uno de los miembros del grupo realiza una parte del trabajo y desconoce las aportaciones de los compañeros hasta llegar al resultado final. En este tipo de práctica no hay corresponsabilidad ni tampoco enriquecimiento de cada uno de los participantes. En estos casos las nuevas formas de aprendizaje, que debería ser cooperativo, se orientan única y exclusivamente a sumar partes individuales de tareas que componen el todo del trabajo, pero sin llegar a un aprendizaje cooperativo real, en el que según Jordán (2006):

- Todos los miembros han de sentirse responsables del éxito de sus compañeros.

- Los miembros han de estar co-implicados en una tarea común motivadora. 
- Todos participan en metas y recompensas comunes.

- Todos tienen la misma oportunidad de alcanzar el dominio de la materia.

- Todos los miembros, incluidos los menos capaces o los minoritarios, han de percibirse individual y mutuamente como poseedores de un similar status.

- Los estudiantes han de tener muy claros cuáles son los objetivos que el profesor desea de su trabajo, tanto en el plano académico como en el conductual y actitudinal.

- Tiene que haber armonía entre la responsabilidad individual y la grupal respecto al producto final.

Todos los informes de seguimiento del progreso de la creación del EEES, preparados por redes de universidades o de estudiantes, afirman que el proceso de cambio se ha retrasado especialmente en un aspecto: en la renovación de los métodos de enseñanza-aprendizaje (Salaburu, Haug y Mora, 2011). El cambio en las maneras de enseñar y aprender resulta ser el más lento. El tema es aún más preocupante porque deja constancia de que no se aprovechan las oportunidades que ofrecen las nuevas tecnologías de información y comunicación. Aun así, existen "buenas prácticas" que marcan las principales líneas de innovación en los métodos, en la evaluación y en todo el proceso de enseñanza-aprendizaje, en general.

Tabla 1. Tabla 1. Evolución del proceso de enseñanza/aprendizaje en la universidad.

\begin{tabular}{|c|c|c|}
\hline & AHORA & ANTES \\
\hline \multirow{3}{*}{ Aprendizaje } & construcción activa & dar información \\
\hline & conexiones & carácter jerárquico \\
\hline & contextualizado & descontextualizado \\
\hline Enseñanza & transformación & transmisión \\
\hline Currículo & moldeable & fijo \\
\hline \multirow{2}{*}{ Tareas } & auténticas & aisladas \\
\hline & conjunto de representaciones & materiales secuenciados \\
\hline \multirow{3}{*}{ Mediación social } & comunidad de aprendizaje & individual \\
\hline & colaboración & competición \\
\hline & discurso & recitación \\
\hline Herramientas & $\begin{array}{l}\text { uso interactivo e integrado de los } \\
\text { ordenadores }\end{array}$ & papel y lápiz \\
\hline \multirow{2}{*}{ Evaluación } & basada en actuaciones & pruebas de rendimiento \\
\hline & carpetas individuales (portfolio) & test estandarizados \\
\hline
\end{tabular}

Fuente: Marcelo (2001)

\section{3.- EL SISTEMA DE ADMISIÓN DE ESTUDIANTES}

No hay que olvidar un primer paso que sería el sistema de admisión de estudiantes (a pesar de que no forma parte de la metodología, condiciona todo el proceso posterior). Parece cada vez más importante crear múltiples puntos de entrada y de salida, con varias vías de acceso (no solamente una, centrada en los resultados académicos en la enseñanza secundaria) y con validación de lo ya aprendido. Esta exigencia es clave para el desarrollo de auténticas estrategias de aprendizaje a lo largo de la vida en las universidades españolas, las cuales están bastante retrasadas lo que no permite reorientar todo el sistema de educación superior en esta dirección (Salaburu et al., 2011). En varias universidades de Europa se han creado pruebas de acceso que miden no solamente los resultados académicos anteriores del candidato, sino también su potencial futuro, teniendo en cuenta sus aptitudes en el campo de estudios al que quiere acceder. 


\section{COMBINACIÓN DE MÉTODOS DE ENSEÑANZA-APRENDIZAJE}

Otro aspecto necesario sería la combinación de una mayor variedad de métodos de enseñanzaaprendizaje. España ha ido abandonando, lentamente, el uso casi exclusivo del modelo de clases magistrales, a pesar de que numerosas investigaciones internacionales habían demostrado su mediocre eficacia. Se recomienda el uso de un amplio abanico de métodos de aprendizaje que, gracias a las TIC, nunca ha sido tan variados (Salaburu et al., 2011):

1) Cursos presenciales o no, en muchos casos en idioma extranjero.

2) Uso adecuado de las TIC como medio de docencia y de auto-aprendizaje.

3) Trabajos de investigación, informes, proyectos, tesinas o tesis, solo o en equipo.

4) Resolución de problemas concretos y complejos (es decir multidisciplinares), solo o en equipo.

5) Prácticas externas en empresas u otras entidades administrativas, culturales o sociales.

6) Actividades formativas extra-curriculares, como la participación en actividades de consultoría (como las junior enterprises o los "clubes de inversores" que organizan los colectivos estudiantiles de muchos países) o la gestión de tareas humanitarias, sociales, etc.

7) Tutoría y orientación personalizada para cada estudiante (abarcando todos los campos formativos, y no solamente los relacionados con el aspecto académico).

Uno de los retos del EEES es desarrollar métodos didácticos que permitan la adquisición de conocimientos, destrezas y competencias y que se pueda evaluar en qué medida se cumplen los objetivos de aprendizaje fijados (los learning outcomes) (Salaburu et al., 2011). Para ello se recomienda utilizar, además de las horas lectivas tradicionales, una mayor variedad de métodos de enseñanza y aprendizaje, presenciales o basados en el uso de las TIC, individuales o de grupo, etc. De esta manera, se pueden satisfacer las necesidades de estudiantes con perfiles cada vez más diversos, como ocurre con el aumento del aprendizaje a lo largo de la vida. Fijar objetivos del aprendizaje basados en un conjunto de conocimientos, destrezas y competencias es un elemento importante para diseñar currículos y métodos no tradicionales y más adaptados al papel estratégico que deben asumir las universidades en la formación de estudiantes.

Los propios estudiantes, en un estudio realizado en la Universidad de Castilla-La Mancha, señalan que la carga de trabajo diario se ha incrementado considerablemente con las nuevas metodologías y critican la acumulación de trabajos que hay que realizar simultáneamente en distintas asignaturas, debido a una mala planificación o coordinación (Rincón Igea y González Geraldo, 2010). Consideran también que el peso de los trabajos debería ser mayor en la evaluación final de la asignatura.

Señalan también el uso excesivo de proyecciones con el programa power-point hasta tal punto que en ocasiones "cuando no funciona el cañón, no hay clase" (Rincón Igea y González Geraldo, 2010, 70).

Con respecto a los trabajos en grupo, los estudiantes destacan ventajas e inconvenientes. La principal ventaja consiste en que "cuatro cabezas piensan más que una". Entre los inconvenientes señalan la pérdida de tiempo para el estudio individual, la dificultad de compaginar horarios y coincidir con los otros miembros del grupo y no en último lugar, el nivel bajo de conocimientos o la poca dedicación de algunos estudiantes del grupo (Rincón Igea y González Geraldo, 2010).

Antes de finalizar este apartado, volvemos a hacer hincapié en la participación activa de los estudiantes. La planificación ya no se orienta solo hacia los contenidos, sino también hacia los estudiantes y sus procesos de adquisición y construcción de conocimiento. Incluso las teorías de aprendizaje más centradas en actividades de enseñanza como las teorías clásicas de estímulo y respuesta, las contemplan al menos en la participación activa de los sujetos en el proceso de vincular estímulos y comportamientos (Huber, 2008). Los estudiantes tienen que vincular activamente los hechos relevantes. 
Los métodos adecuados para el EEES deberían aunar componentes didácticos que respondan a tres retos principales: preparar a los jóvenes para desempeñar su papel en el mundo del trabajo a través de la adquisición de conocimientos y de varias competencias, participar como ciudadanos activos en una sociedad democrática y poder asumir sus responsabilidades personales (Huber, 2008).

Los métodos para un aprendizaje activo, situado, autorregulado, constructivo y social sitúan tanto a los estudiantes como a los profesores ante la dificultad común de que se requieren algunas condiciones previas, que el principiante debería poseer para poder aprender con estos métodos. Por ejemplo, si los estudiantes no han desarrollado ya destrezas de aprendizaje activo, necesitarán ser guiados en todo el proceso.

Algunos de los métodos más recomendados son los siguientes:

1) Métodos por proyectos

2) El método de aprendizaje basado en problemas

3) Los métodos de enseñanza y aprendizaje mutuos

Este último método se basa en cuatro principios (Huber, 2008, 76-77):

- ayudar a los estudiantes con estrategias adecuadas de aprendizaje

- estimular el que los estudiantes se enseñen los unos a los otros

- reconocer y retroalimentar los rendimientos, no de los miembros individuales, sino del conjunto social del equipo

- negociar reglas e iniciar procesos de reflexión

Para el área de enseñanza-aprendizaje de lenguas (L1, L2 y LE) recomendamos consultar los métodos incluidos en el Diccionario de términos clave de ELE (Instituto Cervantes, 1997-2015).

\section{EVALUACIÓN FORMATIVA DE LOS ESTUDIANTES}

La evaluación formativa es la que va más allá de la calificación final de la asignatura. La evaluación formativa pretende comprobar si los estudiantes han adquirido, además de los conocimientos relacionados con la materia, las distintas competencias y destrezas en los objetivos de cada currículo. La evaluación, además, debe proporcionar oportunidades de mejora.

En muchos países de Europa los estudiantes se quejan de que esta evaluación es la que mayor retraso tiene en el proceso renovador. Entre los ejemplos de "buenas prácticas" en este ámbito se pueden mencionar al menos las dos siguientes (Salaburu, Haug y Mora, 2011):

- La creación de comisiones de exámenes, que toma una decisión colectiva, evitando así la calificación individual de un solo docente.

- La participación de observadores externos en los exámenes, que procedan de otras universidades y emitan una opinión sobre la idoneidad de los exámenes y de los resultados.

Hemos señalado anteriormente que los propios estudiantes consideran que la carga de los trabajos debería tener un mayor porcentaje en la nota final de la asignatura. Al no recibir este reconocimiento, se sienten cada vez menos motivados para el estudio constante y para la realización de trabajos. Algunas de las opiniones apuntan a que la sobrecarga de trabajos puede producir agobio, estrés y ansiedad. Por eso, llegan a opinar que "sale más rentable la opción antigua porque no vas perdiendo nota en los trabajos pequeños" (Rincón Igea y González Geraldo, 2010, 72). La opinión que predomina, recogida en el mismo estudio, consiste en que la evaluación final no debe limitarse solo a un examen, además de que algunos tienen verdadero pavor al examen final, sino que se debe eliminar materia a través de parciales y de trabajos realizados de forma continua.

Hay quienes dudan de la objetividad de la evaluación con el sistema de ECTS y sospechan que se produce cierta arbitrariedad. Señalan como ejemplo de subjetividad las guías docentes en las que los profesores indican que se valora la participación en clase. 


\section{LA TUTORÍA UNIVERSITARIA}

En el EEES se pone de manifiesto la importancia de la orientación como un elemento clave en la formación universitaria. La tutoría académica o la acción tutorial es el instrumento que permite realizar este proceso de orientación. Consiste en un proceso de acompañamiento de carácter formativo, orientador e integral desarrollado por docentes universitarios. Tiene como finalidad facilitar a los estudiantes todas las herramientas y la ayuda necesarias para conseguir con éxito tanto los objetivos académicos, como los personales y profesionales, que les plantea la universidad.

Las tutorías son un método de aprendizaje integrado en los planes de estudios de las nuevas titulaciones y tienen un papel fundamental para individualizar, supervisar y evaluar el trabajo autónomo del estudiante. Por ello, por regla general son de asistencia programada y obligatoria y se computan como horas de trabajo presencial del estudiante.

El profesor actúa como mediador y monitor de las actividades de aprendizaje del alumnado para alcanzar las competencias (Madrid Manrique, 2012). Se incorporan más sesiones de tutoría y apoyo, pero esto requiere una reducción del número de estudiantes para posibilitar la individualización de la enseñanza y la tutorización del aprendizaje (Madrid Manrique, 2012). Todo esto supone una renovación metodológica de la actividad docente, basada en una educación centrada en el alumno, donde el profesorado ejerce nuevas funciones con un enfoque de la enseñanza por competencias o por objetivos formulados como resultados de aprendizaje.

\section{Características de la tutoría universitaria}

Las características básicas de la tutoría universitaria se pueden resumir de la siguiente manera (Rodríguez Espinar, 2008 [2004]):

- Es una acción docente de orientación dirigida a impulsar y facilitar el desarrollo integral de los estudiantes en su dimensión intelectual, afectiva, personal y social, en línea con un planteamiento de calidad desde la perspectiva del estudiante.

- La tutoría contribuye a personalizar la educación universitaria. La enseñanza en la universidad pretende que las personas construyan y maduren unos conocimientos y unas actitudes. Teniendo en cuenta que el aprendizaje es una evolución personal de cada alumno y que la característica fundamental de la universidad del siglo XXI es el incremento en la heterogeneidad del alumnado (en cuanto a la edad, expectativas, participación laboral durante los estudios, etc.), la tutoría debe facilitar el seguimiento académico individualizado de los estudiantes en la planificación y desarrollo de su itinerario de formación.

- La atención al estudiante constituye un elemento clave de calidad. La ratio de alumnos convierte la tutoría en un recurso clave y sin alternativa. Así, en los países donde ha predominado el modelo únicamente de servicios de orientación, independientes de la actividad académica, no puede llegarse a atender al conjunto de estudiantes, de manera que su actuación se reduce a la información más o menos extensiva, o bien, al asesoramiento intensivo, pero minoritario.

- La tutoría canaliza y dinamiza las relaciones del alumnado con los diferentes segmentos de atención al estudiante, tanto de carácter administrativo (facilitando el acceso y la interpretación de la información), docente (contribuyendo a la comprensión del currículum formativo de los estudios y del significado y demandas de las diferentes materias), organizativo (favoreciendo la participación, etc.) y de servicios (de orientación, culturales, etc. a través de favorecer su conocimiento y el encuentro entre ambos). De este modo, se convierte en un garante del uso adecuado de los diferentes recursos curriculares y extracurriculares que la institución pone a su alcance.

- Al facilitar este último objetivo, la tutoría permite la integración activa del estudiante en la institución. No solo porque facilita el acceso a la información, de una forma crítica y constructiva, sino también porque estimula la implicación y participación en todos los niveles organizativos. 


\section{Dimensiones y niveles de intervención en la tutoría}

A continuación resumimos las dimensiones y niveles de intervención en el desarrollo personal del estudiante que se producen en las tutorías (Rodríguez Espinar, 2008 [2004]), 29):

- Dimensión intelectual y cognitiva: incluye desarrollar aspectos que potencien la capacidad de enfrentarse a situaciones problemáticas $\mathrm{y}$ a su resolución, a la adecuada toma de decisiones, la rentabilización de los aprendizajes, el aprender a aprender...

- Dimensión afectivoemotiva: potenciar el dominio de las habilidades sociales, el autoconocimiento, la elaboración de un autoconcepto positivo...

- Dimensión social: esta dimensión abarca conseguir la integración en un grupo y la participación activa en el mismo en un contexto de cooperación.

- Dimensión profesional: contribuir a tomar conciencia de su propio itinerario curricular, a la elección de un proyecto profesional y el acceso a la profesión.

$\mathrm{La}$ atención a estas dimensiones presenta diferencias considerables en función de la tradición en cada país. Normalmente, se aplican modelos integrales compuestos de tres niveles de intervención en las tutorías (Rodríguez Espinar, 2008 [2004]):

- La tutoría de materia consiste en orientar al estudiante sobre contenidos disciplinares que el profesor expone en clase o sobre los trabajos relacionados con la asignatura.

Tabla 2.- Tipos de tutorías según criterios.

Criterios Tipos de tutoría

En relación con los servicios de orientación

En cuanto al contenido de las tutorías

En cuanto a la figura del tutor

Con relación al tiempo

En cuanto a los

destinatarios Tutoría grupal: posibilita la acción sobre más alumnos en menor tiempo, al tratar temas comunes, intercambiar experiencias...

Modelo tutorial puro: el programa de tutorías funciona de modo independiente de los servicios de orientación de la institución.

Modelo mixto: el modelo tutorial se organiza con apoyo de los servicios de orientación.

Tutoría de materia: se ocupa de orientar al estudiante sobre temas relativos a los contenidos disciplinares que el profesor expone o presenta en clase, o sobre temas de trabajos relacionados con la materia.

Tutoría de prácticas: tiene como finalidad el proporcionar al alumno en prácticas habilidades y herramientas para desarrollar con competencia profesional las prácticas.

Tutoría de proyecto: asesorar y orientar todo el trabajo de proyecto del alumno (relativo a demandas de final de algunas carreras).

Tutoría de asesoramiento personal: corresponde a una tutoría especializada para el tratamiento o la intervención ante determinadas circunstancias personales de algunos estudiantes $y$ es responsabilidad de profesores expertos en la intervención psicopedagógica, o bien consiste en el uso de servicios especializados de la propia universidad a través de la derivación que pueda hacer el tutor académico o el tutor de carrera.

Profesor-tutor: la tutoría es asumida por el profesor.

Tutoría de iguales (peer tutoring): los alumnos de cursos superiores son los encargados de orientar y asesorar a los compañeros, de forma exclusiva o como acción complementaria.

Tutoría de curso: se refiere al seguimiento del alumno en un tramo de su proyecto formativo.

Tutoría de carrera o de itinerario académico: se refiere a un seguimiento del estudiante a lo largo de los estudios universitarios, en cuestiones generales relativas a los itinerarios curriculares, a la adaptación a la vida universitaria, a la mejora del rendimiento o a las salidas profesionales.

Tutoría individual: acción personalizada, útil para tratar aspectos personales de carácter individual. 
- La tutoría de carrera o de itinerario académico es en la que se realiza un seguimiento del estudiante a lo largo de sus estudios universitarios, atendiendo cuestiones generales de los itinerarios curriculares, la adaptación al ámbito universitario, la mejora del rendimiento académico o las salidas profesionales.

- La tutoría de asesoramiento personal corresponde a una tutoría especializada para tratar circunstancias personales de algunos estudiantes y es responsabilidad de profesores expertos en intervención psicopedagógica. En ocasiones, el tutor puede derivar al alumno a usar servicios especializados de la universidad.

\section{Tipos de tutoría}

Hemos de mencionar también los tipos de tutorías (Tabla 2). Para ello presentamos un cuadro de Rodríguez Espinar (2008, 31-33) que resume los tipos de tutorías que existen actualmente, clasificados en función de diferentes criterios, contemplados en su definición. De todas formas, hay que tener en cuenta que estos tipos no son excluyentes, sino más bien complementarios.

\section{Roles y competencias del profesor tutor}

Para poder desempeñar la labor de profesor tutor se necesitan unas competencias que corresponden a determinados roles que esta figura tiene en la acción tutorial. Siguiendo a Rodríguez Espinar $(2008,64)$, el profesor tutor debería tener las siguientes competencias (Tabla 3):

- competencias a nivel cognitivo: saber (conocimientos) y saber hacer (comp. prácticas)

- competencias de carácter social y de relación: saber estar (actitudes y comportamientos de tipo participativo) y saber ser (actitudes y comportamientos de tipo personal)

Tabla 3.- Competencias del profesorado.

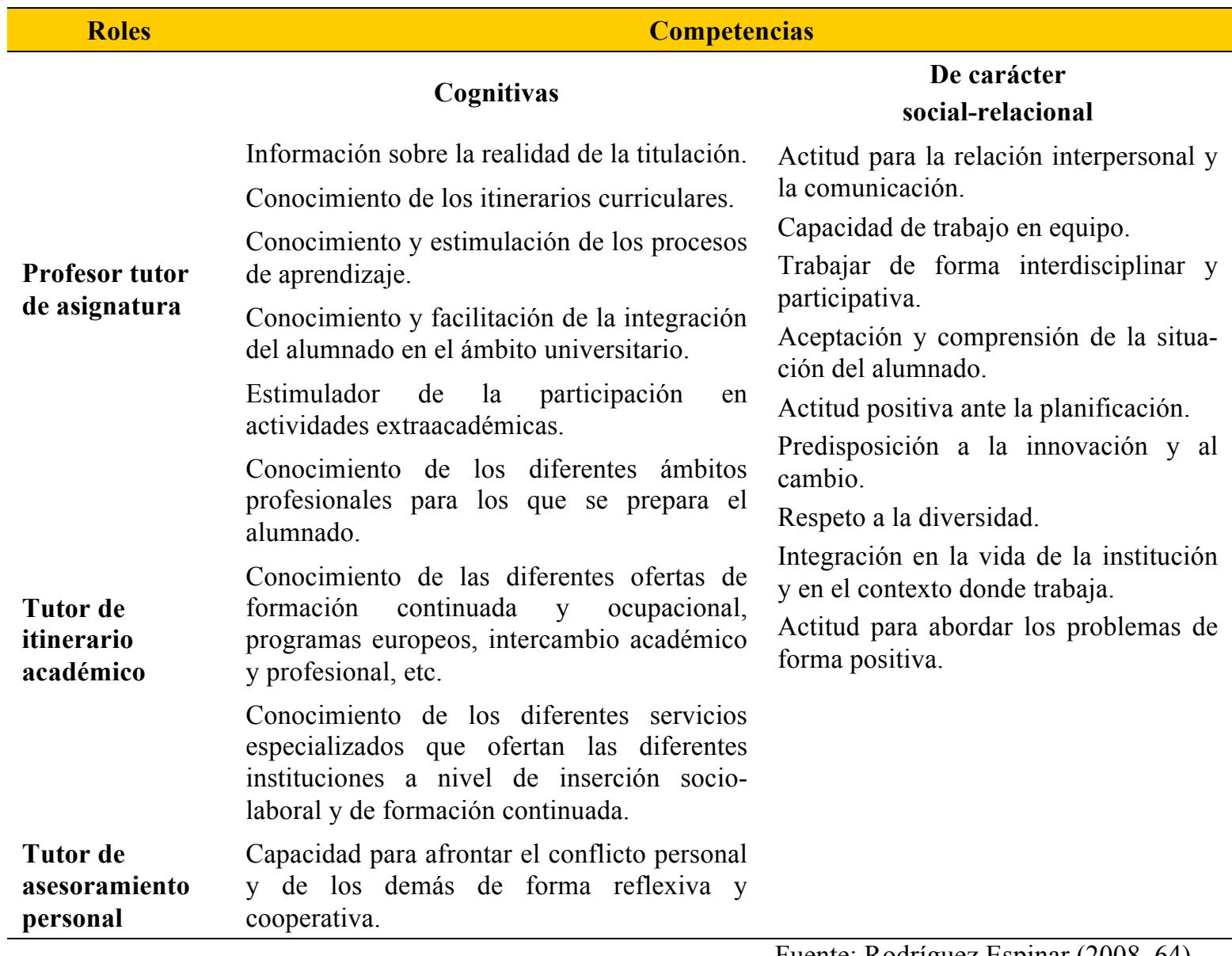

Fuente: Rodríguez Espinar (2008, 64). 


\section{Reflexión final sobre las tutorías}

Según la opinión de los propios estudiantes (Rincón Igea y González Geraldo, 2010), las tutorías resultan de difícil acceso debido a los solapamientos horarios con clases de asignaturas obligatorias. El criterio horario está centrado en el profesor.

En ocasiones, los profesores no están presentes, pero en general, la acogida y la disposición del profesorado es muy buena.

Por último, hemos de recordar que la tutoría surge para dar respuestas a las nuevas necesidades de los estudiantes y de la universidad y que los planes de acción tutorial se inscriben entre las medidas de calidad de la universidad como institución.

Sin pretender abarcar todos los aspectos de la renovación metodológica en este apartado (algunos se detallan en otros apartados de este proyecto), podríamos resumir que el aprendizaje cooperativo, la incorporación de las TIC, la tutoría universitaria, como las bases de la nueva metodología en el Espacio Europeo, junto con los nuevos roles profesor/alumno, entre otros factores, aún siguen siendo un reto por conseguir, no solo por la concienciación del propio profesorado, como agentes directos del cambio y de sus posibilidades de éxito, sino también por la falta de recursos (elevadísimas ratios profesor/alumno por aula, formación del profesorado en TIC, etc.) y de inversión propia de cada país. En cualquier caso, todo ello unido a la vertiginosa situación de crisis económica internacional está siendo una limitación común para el logro de la transformación real del modelo de enseñanza universitario.

\section{Una reflexión crítica sobre el Espacio Europeo de Educación Superior}

Ante los cambios ya implantados en el EEES y después de numerosos estudios y análisis del nuevo sistema educativo, frutos de la preocupación de distintos sectores relacionados con el ámbito de la enseñanza, no han faltado las opiniones críticas a esta convergencia europea.

Antes de resumir las críticas más frecuentes, nos parece conveniente recordar algunos aspectos fundamentales. Por una parte, este proceso de renovación universitaria en el marco del EEES es de carácter voluntario para los países miembros. Si se decide implantarlo, se hace bajo la responsabilidad principal de cada gobierno, así como de sus instituciones de educación superior. Las únicas reglas "obligatorias" (que derivan de la decisión libre de cada país de adherirse al EEES) son de naturaleza formal o estructural y conciernen a la secuencia de titulaciones (o cualificaciones), la definición de cada nivel en términos (flexibles) de créditos ECTS y la expedición del Suplemento al Diploma (Salaburu et al., 2011). Por otra parte, no se trata de conseguir una uniformización de la oferta formativa, ni de la metodología, sino que incluso se recomienda la diversificación. Lo que se pretende es que sea más fácil seleccionar la universidad y los estudios que mejor le convengan a cada uno.

Hemos de resaltar también que la normativa en ocasiones parece excesivamente reguladora, lo que ha provocado que las universidades perciban el proceso de Bolonia más bien como una exigencia del Ministerio de Educación y no como una oportunidad para adaptarse al EEES. En este sentido, coincidimos con Salaburu et al. $(2011,228)$ quienes señalan que "no podremos realizar cambios con perspectiva europea, si la referencia de cambios que asumimos es cerrada y exclusivamente española".

Asimismo, hayque admitir que la universidad española nunca ha sido tan fuerte como hoy, sobre todo en el campo de la investigación, y que necesita no obstante modernizarse profundamente, sobre todo en el campo de la docencia y la organización interna, para que pueda desempañar plenamente su papel en la nueva edad del conocimiento y de la globalización y en el nuevo espacio europeo (Salaburu et al., 2011).

Los problemas a los que tiene que enfrentarse la universidad española, se pueden resumir, en líneas generales, en la necesidad de aumentar la relevancia social de la formación y de la investigación, la eficiencia de la educación superior, la autonomía de las universidades y su diferenciación, así como el reto de aumentar su prestigio internacional.

En el marco de la estrategia de Lisboa y en el Comunicado de la Comisión de las Comunidades Europeas, en Bruselas en 2005 (citado por Salaburu et al., 2011), se indica que los tres 
vértices del triángulo del conocimiento son la educación, la investigación y la innovación y la universidad debe tener un papel decisivo en cada uno de estos ámbitos. Se señala también que los tres retos principales en la educación superior son: conseguir un nivel de calidad mundial, mejorar la gobernanza e incrementar y diversificar la financiación. En nuestra opinión, todo esto no se ha conseguido todavía, por lo que sigue siendo válido y actual.

En el mismo documento se indican los siguientes puntos débiles:

1) Uniformidad estudiantes.

Tendencia a la uniformidad respecto a los programas formativos, los métodos y el perfil de los

2) Fragmentación y aislamiento

Se trata de la fragmentación que todavía existe por países e incluso de un mismo país. Se siguen usando normativas distintas en lenguas distintas, lo que no facilita la comprensión a escala internacional.

\section{4) Una reglamentación excesiva}

La excesiva reglamentación entorpece la modernización de las universidades y hace disminuir su eficacia. La escasa flexibilidad de las normas de admisión y de reconocimiento de títulos constituye un obstáculo para el aprendizaje permanente y la movilidad. Las universidades no pueden reaccionar rápido a los cambios que se producen en su entorno, porque cuando el cambio pasa invariablemente por la legislación, las reformas son forzosamente escasas, incómodas y uniformes.

\section{5) Una financiación insuficiente}

Se pueden señalar dos grandes déficits de inversión:

- en el ámbito de la investigación, el gasto de los países de la Unión Europea se sitúa en el $1,9 \%$ del PIB, mientras que en los Estados Unidos, Japón y Corea del Sur alcanza casi el $3 \%$, una diferencia que se explica por el nivel de inversión de la industria en estos países, que es mucho mayor;

- en el ámbito de la enseñanza superior, los países de la Unión gastan por término medio solo un $1,1 \%$ del PIB, una cifra similar a la de Japón, pero muy inferior a las de Canadá $(2,5 \%)$, los Estados Unidos $(2,7 \%)$ y Corea del Sur $(2,7 \%)$. Esta diferencia obedece casi enteramente a que los niveles de inversión de la industria y los hogares son mucho más bajos en nuestro continente. Para igualar a los Estados Unidos, Europa tendría que destinar cada año 150000 millones de euros suplementarios a la enseñanza superior.

En el citado documento de la Comisión se señala también que en términos estratégicos, las consecuencias de una financiación insuficiente y de la dependencia de los fondos públicos no se limitan solo al empobrecimiento del sector de la enseñanza superior, sino que se traducen en otras carencias, más o menos pronunciadas según el país de que se trate: bajos niveles de matriculación, imposibilidad de responder satisfactoriamente a la demanda, incapacidad de preparar a los estudiantes para el mercado de trabajo europeo, escasez de puestos de trabajo para el personal docente o investigador o dificultades a la hora de atraer y retener los talentos más brillantes.

Algunos autores denuncian la concepción mercantilista de la educación. Consideran que el actual modelo educativo está basado en unas competencias propuestas y seleccionadas a partir de las demandas económicas y empresariales de los grupos económico-sociales dominantes. En la misma línea López Galera (2012) afirma que se ha adoptado una concepción funcionalista, pragmática y mercantilista de la universidad en respuesta a las demandas del mercado laboral (Madrid Manrique, 2012). Palomero y Torrego (2005) cuestionan si trabajamos actualmente para conseguir una mayor calidad educativa en los estudios superiores o si se trata simplemente de una política de mercado,

Escudero $(2012,75)$ destaca entre sus críticas la actual orientación hacia el producto que predomina sobre el modelo procesual y el énfasis en el conocimiento procedimental en detrimento del conceptual: 
... valora más la acción visible que el pensamiento, más los resultados que los procesos, más los métodos o el cómo hacer que las bases sustantivas (contenidos, teorías, modelos de explicación y comprensión) en las que se justifican y apoyan.

Tal como señala Madrid Manrique (2012) la sustitución de la concepción tradicional de la enseñanza centrada en los contenidos de las materias por una enseñanza orientada hacia la adquisición de competencias específicas ha recibido también numerosas críticas (Pérez Gómez, 2007; Madrid y Hughes, 2012). En sus reflexiones críticas, hace hincapié en los siguientes aspectos:

1) Excesiva ambigüedad y falta de claridad en los conceptos.

Señala la dificultad de diferenciar términos como competencia, capacidad u objetivos generales y por consiguiente, de aplicarlos a la práctica o de evaluarlos.

2) Enfoque reduccionista.

El hecho de programar el proceso formativo por competencias ha llevado a algunos autores a considerarlo un enfoque demasiado reduccionista, parcial y unilateral (Moreno y Soto, 2005).

3) Falta de un tratamiento secuenciado, coordinado y colectivo de la inclusión y desarrollo de las competencias.

La propuesta de competencias (como mero listado de capacidades) se enmarca en un ámbito académico sin cambiar en lo fundamental y "supone un parche que introduce un simple cambio de lenguaje y de collar"(Madrid Manrique, 2012, 25).

4) Falta de precisión y delimitación en los criterios de evaluación.

Con frecuencia, se mezclan criterios de evaluación con actividades concretas por lo que es recomendable definir y delimitar los apartados de metodología y de evaluación dentro del desarrollo de cada competencia.

Resumiendo, existe una preocupación por la validez que cobraría la cantidad en lugar de la calidad, los resultados en lugar del proceso, los intereses económicos, la competitividad y las demandas del mercado en lugar de la formación integral del individuo.

Se han desarrollado múltiples actividades de difusión de los conceptos principales de la reforma en el EEES, pero la comunidad universitaria necesita una mayor profundidad de análisis, debate y crítica de este proceso tan crucial para toda la sociedad.

\section{8.- REFERENCIAS}

Arranz, P. (2008). Innovación curricular: aulas inclusivas. Anuario de Pedagogía, 3, 143-152.

Contreras Conzález, L. C. (2007). El marco de la Convergencia Europea: los créditos ECTS. En Fonseca Mora y Aguaded Gómez (eds.), Enseñar en la Universidad. Experiencias y propuestas de docencia universitaria (pp. 3-12).La Coruña: Netbiblo.

Escudero Muñoz, J. M. (2012). La elaboración de las titulaciones universitarias en el contexto del Espacio Europeo de Educación Superior: condiciones, procesos, resultados e interrogantes. En J. Bautista Martínez Rodríguez (ed.), Innovación en la universidad. Prácticas, políticas y retóricas (pp. 53-84). Barcelona: Graó.

Huber, G. L. (2008). Aprendizaje activo y metodologías educativas. Revista de Educación, número extraordinario 2008, pp. 59-81.

Imbernón, F. (1999). Los retos de la universidad del futuro. Forum Telemático Ariadna. En ftp://ariadna.d5.ub.es/pub/forum

Instituto Cervantes (1997-2015). Diccionario de términos clave de ELE. Disponible en: http://cvc.cervantes.es/ensenanza/biblioteca_ele/diccio_ele/default.htm

Jordán, J. A. (2006). La interculturalidad en la escuela. Revista Interuniversitaria de formación del profesorado, 25, 71-84.

López Galera, N. (2012). Prólogo: Bolonia sin filosofía. En J. Bautista Martínez Rodríguez (ed.), Innovación en la universidad. Prácticas, políticas y retóricas (pp. 11-17). Barcelona: Graó. 
López Yánez, J. y Rodríguez López, J. M. (2007). Estudio de casos como metodología centrada en el aprendizaje. En Fonseca Mora y Aguaded Gómez (dir.), Enseñar en la Universidad. Experiencias y propuestas de docencia universitaria (pp. 24-36). La Coruña: Netbiblo.

Madrid Manrique, M. (2012). La aplicación del crédito europeo en Educación artística. Granada: Editorial GEU.

Madrid, D. y Hughes, St. (2012). Competences and foreign language teacher education in Spain. En M. L. Pérez Cañado (ed.), Competency-based Language Teaching in Higher Education (pp. 63-75). Amsterdam: Springer.

Marcelo, C. (2001). El proyecto docente: una ocasión para aprender. En J. Alonso Tapia y A. García-Valcárcel (coord.), Didáctica universitaria (pp. 45-77). Madrid: La Muralla.

McCombs, B. y Whisler, J. S. (2000). La clase y la escuela centrada en el aprendiz. Estrategias para aumentar la motivación y el rendimiento. Barcelona: Paidós.

Moreno Moreno, P. y Soto Martínez, G. (2005). Una mirada reflexiva y crítica al enfoque por competencias. Educar, 35, 73-80.

Palomero Pescador, J. E. y Torrego Egido, L. (2005). "Europa y calidad docente ¿Convergencia o reforma educativa" (presentación del XI Congreso de Formación del Profesorado: Europa y calidad docente ¿Convergencia o Reforma Educativa?, celebrado en Segovia, del 17 al 19 de febrero de 2005). Disponible en www.aufop.org/xi-congreso/PresentaRIFOP.doc

Pérez Gómez, A. I. (2007). Las competencias básicas: su naturaleza e implicaciones pedagógicas (Cuaderno de Educación n. ${ }^{\circ}$ 1). Santander: Consejería de Educación de Cantabria.

Rincón Igea, B. del y González Geraldo, J. L. (2010). La voz de los estudiantes en el EEES: Valoraciones sobre la implantación de los ECTS en la UCLM. Revista Docencia e Investigación, 20,59-85.

Rodríguez Espinar, S. (2008 [2004]). Manual de tutoría universitaria. Barcelona: Ediciones Octaedro.

Salaburu, P., Haug, G. y Mora, J. G. (2011). España y el proceso de Bolonia. Un encuentro imprescindible. Madrid: Academia Europea de Ciencias y Artes.

Tomás, M. (2000). La gestión académica de las universidades: lugar de encuentro entre facultades y departamentos. En Seminario Internacional EAIR UPC, Barcelona. 